\title{
Diagnóstico da ocorrência de aranhas do gênero Loxosceles Heineken e Lowe, 1832 (Araneae, Sicariidae) no município de União da Vitória, Paraná
}

\author{
Marta Luciane Fischer ${ }^{1 *}$ \\ Sergio Bazilio ${ }^{2}$ \\ Thalita Verginia Batista dos Santos ${ }^{2}$ \\ Cristina Brandes Grosskopf ${ }^{2}$ \\ ${ }^{1}$ Núcleo de Estudos do Comportamento Animal, Departamento de Biologia \\ Pontifícia Universidade Católica do Paraná \\ Av. Silva Jardim, 1664/1101, CEP 80250-200, Curitiba - PR, Brasil \\ ${ }^{2}$ Colegiado de Ciências Biológicas, Faculdade de Filosofia, Ciências e Letras de União da Vitória \\ *Autor para correspondência \\ marta.fischer@pucpr.br
}

Submetido em 24/07/2008

Aceito para publicação em 10/11/2008

\section{Resumo}

Esse trabalho tem por objetivo realizar um diagnóstico comparado da ocorrência de populações de Loxosceles sp. (aranha-marrom) no município de União da Vitória, Paraná. Foram inspecionados substratos naturais e antrópicos presentes no intra- e peridomicílio da área urbana e rural. Loxosceles intermedia MelloLeitão, 1934 ocorreu em 57\% das residências, porém não ocorreu homogeneamente entre os diferentes bairros e substratos e foi menos freqüente do que outras espécies de aranhas encontradas no mesmo habitat. A pequena notificação de loxoscelismo pode ser devida à pequena população de aranhas-marrom em ambientes domiciliares. Apesar de encontrar substratos e condições climáticas aparentemente adequadas no ambiente antrópico, o crescimento de populações de L. intermedia pode ser limitado pela presença de outras espécies de aranhas.

Unitermos: ambiente antrópico, aranha-marrom, fauna sinantrópica, Loxosceles intermedia, Loxoscelismo

\section{Abstract}

Diagnosis of the occurrence of the genus Loxosceles Heineken and Lowe, 1832 (Araneae, Sicariidae) in the municipal district of União da Vitória, Paraná. This paper aims to carry out a comparative evaluation of the populations of Loxosceles spp. (brown spiders) found in the municipal district of União da Vitória, Paraná. Natural and anthropic substrata were inspected in the intra- and peridomicile in urban and rural areas. Loxosceles intermedia Mello-Leitão, 1934 was present in 57\% of the residences. It was not randomly distributed among the neighborhoods or the substrata and was less frequent than other species of spiders found in the same habitats. The few records of loxoscelism may be due to the small population of brown spiders in domestic habitats. Notwithstanding the seemingly adequate climatic and substratic conditions, the population growth of L. intermedia might be limited by the presence of other species inside and around houses.

Key words: anthropic environment, synanthropic fauna, brown spider, Loxosceles intermedia, loxoscelism 
O gênero Loxosceles Heinecken \& Lowe, 1832 é representado por cerca de 100 espécies com potencial sinantrópico (Gertsch, 1967). Entretanto, características específicas como hábito mais ou menos generalista e maior ou menor atividade locomotora fora da área da teia podem maximizar a colonização de ambientes antrópicos, resultando em padrões atípicos como os apresentados por Loxosceles intermedia Mello-Leitão, 1934 em Curitiba (Fischer e Vasconcellos-Neto, 2005). Devem ser consideradas, porém, características bióticas e abióticas do ambiente (Marques-da-Silva e Fischer, 2005). Caso as condições climáticas locais sejam instáveis e o ambiente externo apresente extremos de umidade e temperatura, o tamanho das populações dessas aranhas, que encontram condições favoráveis no ambiente antrópico, pode ser limitado (Fischer e Vasconcellos-Neto, 2003). A presença de grande quantidade de substratos, que favorecem a residência de inúmeras aranhas e que apresentem propriedades de isolantes térmicos que amenizem as variações climáticas, devido a suas propriedades de isolantes térmicos, e de presas similares às do ambiente natural (Fischer et al., 2006) são fatores extremamente importantes para a delimitação do tamanho da população de espécies sinantrópicas. A baixa ocorrência de outras espécies de aranhas no intra- e peridomicílio, principalmente de espécies que poderiam constituir potenciais predadores ou competidores pode ser um fator favorecedor do grande tamanho da população dessas aranhas (Fischer et al., 2006; Fischer e Krechemer, 2007).

Um amplo estudo de diagnóstico das populações de L. intermedia e L. laeta (Nicolet, 1949) foi realizado por Fischer e Vasconcellos-Neto (2005) em substratos presentes no peri- e intradomicílio em Curitiba, oferecendo parâmetros comparativos que possibilitam a elaboração de protocolos para diagnósticos locais.

O município de União da Vitória (26¹3'48"S e $\left.51^{\circ} 05^{\prime} 09^{\prime \prime} \mathrm{W}\right)$, localizado no sul do Paraná, a $237 \mathrm{~km}$ da capital, possui $731,72 \mathrm{~km}^{2}$ e 51.350 habitantes. O clima é subtropical úmido e mesotérmico, sempre com alta umidade relativa do ar, sendo a temperatura média anual de 23,30 $3^{\circ}$ (Maack, 1981; Hort, 1990). O município apresenta poucos registros de acidentes loxoscélicos somando 89 acidentes de 1993 a 2006, quando comparado com municípios menores próximos como São Mateus do Sul (2552'26”S 50²2'58”W que apresenta 243 acidentes no mesmo período (SVS/ SESA, 2007, comunicação pessoal) ou com o município de Curitiba, onde foram registrados 15.655 acidentes de 1993 a 2000 (Marques-da-Silva et al., 2006). Essa diferença pode ser devida uma população relativamente pequena de aranhas do gênero Loxosceles. Parte-se da interpretação de Fischer e Vasconcellos-Neto (2005), segundo a qual L. intermedia é uma espécie generalista que ocupa facilmente diversos substratos em diferentes condições ambientais e que se move com freqüência favorecendo sua dispersão ativa e passiva. Assim, faz-se necessário o diagnóstico no município de União da Vitória a fim de se conhecer a situação atual das populações de aranhas-marrons. Esse diagnóstico poderia orientar a tomada de medidas preventivas, a fim de evitar que a aranha-marrom se torne um problema de saúde pública, como em Curitiba (Marques-da-Silva et al., 2006). Logo, o presente estudo teve como objetivo diagnosticar as populações de aranhas do gênero Loxosceles ocorrentes no município de União da Vitória, Paraná.

O diagnóstico das populações de aranhas do gênero Loxosceles ocorrentes no município de União da Vitória seguiu a metodologia de amostragem, os parâmetros e variáveis e a categorização dos substratos adotados por Fischer e Vasconcellos-Neto (2005). A amostragem em ambiente natural ocorreu no Bosque das Araucárias

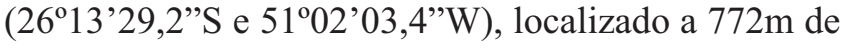
altitude, com área total de $1.181 .550 \mathrm{~m}^{2}$, da qual $20 \%$ corresponde ao bosque propriamente dito.

As amostragens no intra- e peridomicílio foram realizadas em ambiente urbano e rural. Para o ambiente urbano foram realizadas coletas diurnas no período de outubro de 2006 a março de 2007, em três casas por bairro, escolhidas por sorteio após a enumeração das quadras. Residências presentes a 5,10 e $15 \mathrm{~km}$ da área urbana, em cada uma das três vias de saída da cidade, foram consideradas como inseridas na área rural. Devido à pequena quantidade de aranhas na área urbana a análise dos substratos ocupados foi realizada apenas na área rural. As aranhas coletadas foram tombadas na coleção aracnológica do Instituto Butantan (IBSP, curador A. D. Brescovit). 
A amostragem no Bosque das Araucárias totalizou $150 \mathrm{~m}^{2}$ com a inspeção de 44 árvores e 10 troncos caídos, não sendo encontrada nenhuma aranha do gênero Loxosceles ou seus vestígios. No entanto, em habitats antrópicos situados no entorno do bosque (telha de cerâmica), foram encontrados 14 espécimes de L. intermedia (4 fêmeas, 10 jovens) e dois indivíduos de espécies das famílias Amaurobidae e Lycosidae. Esses resultados sugerem que as condições climáticas no ambiente natural, principalmente a alta umidade, não favorecem a colonização de cascas parcialmente desprendidas de árvores, fendas em rochas ou serapilheira, uma vez que as aranhas estão presentes em substratos antrópicos no entorno. Resultados similares foram obtidos por Fischer e Vasconcellos-Neto (2005) em Curitiba, os quais questionaram se a ausência da aranhamarrom nos substratos naturais seria devido a uma migração para os substratos antrópicos ou uma ocupação primária do ambiente urbano. Devido à ausência de vestígios de uma ocupação prévia na natureza, Fischer e Vasconcellos-Neto (2005) consideraram que a hipótese de uma colonização tardia seria mais provável. Este padrão parece se repetir em União da Vitória segundo os dados deste trabalho.

$\mathrm{Na}$ área urbana de União da Vitória, foram inspecionadas 51 residências, em 17 bairros, com 49\% das casas apresentando ocorrência de L. intermedia. No entanto, a distribuição pelos bairros não foi homogênea $\left(\chi_{(16)}^{2}=81,3 ; \mathrm{P}<0,001\right)$, com ocorrência mais freqüente em Navegantes (14,9\%), Rocio (13,5\%) e Nossa Senhora Salete $(12,1 \%)$, caracterizados por maior presença de material de construção depositado no peridomicílio do que nos demais bairros (Centro 0\%, Dona Mercedes 0\%, Bom Jesus 1,3\%, São Bazílio Magno 2,7\%, B. M. Rocha 2,7\%, Cristo Rei 4\%, São Joaquim 4\%, Cidade Jardim 5,4\%, Sagrada Família 5,4\%, Ponte Nova 5,4\%, São Sebastião 6,8\%, São Braz 6,8\%, São Gabriel 6,8\% e São Bernado 8\%). Na área rural, todas as nove casas vistoriadas continham $\mathrm{L}$. intermedia, porém, a distribuição nas três rodovias também não foi homogênea $\left(\chi_{(2)}^{2}=8,3\right.$; $\mathrm{P}<0,05)$, ocorrendo significativamente menos aranhas no Rio Vermelho (21,5\%) do que na Galícia $(40,8 \%)$ e Poço Preto (37,7\%). No entanto, apesar da freqüência de infestação, o número médio de aranhas por casa foi pequeno e mais freqüente no ambiente rural (Tabela 1).
No ambiente urbano, o número de aranhas não diferiu no intra- e peridomicílio, enquanto ocorreram mais aranhas no peridomicílio em ambiente rural (Tabela 1).

TABELA 1: Número médio e total de aranhas amostradas no intra- e peridomicílio da área urbana e rural do município de União da Vitória. As médias foram comparadas através do teste não-paramétrico de MannWhitney e as freqüências totais através do teste do qui-quadrado sendo os valores significativamente diferentes $(\mathrm{P}<0,05)$ acompanhados de letras diferentes.

\begin{tabular}{|c|c|c|c|c|}
\hline & \multicolumn{2}{|l|}{ Urbano } & \multicolumn{2}{|l|}{ Rural } \\
\hline & $\mathrm{N}^{\circ}$ Médio & $\mathrm{N}$ & $\mathrm{N}^{\circ}$ Médio & $\mathrm{N}$ \\
\hline Intradomicílio & $\begin{array}{l}1,2 \pm 0,54 \\
(\mathrm{~N}=23, \text { i.v. }=1-3) \mathrm{a}\end{array}$ & $29 a$ & $\begin{array}{l}8 \pm 3,5 \\
\mathrm{~N}=8, \text { i.v. }=2-13) a\end{array}$ & $46 a$ \\
\hline Peridomicílio & $\begin{array}{l}2,4 \pm 2,4 \\
(\mathrm{~N}=17, \text { i.v. }=1-9) \mathrm{a}\end{array}$ & $41 \mathrm{a}$ & $\begin{array}{l}3 \pm 7 \\
N=9, \text { i.v. }=4-26) b\end{array}$ & $64 b$ \\
\hline Total & $\begin{array}{l}3 \pm 1,6 \\
(\mathrm{~N}=26 ; \text { i.v. }=1-7)\end{array}$ & & $\begin{array}{l}4,4 \pm 8 \\
\mathrm{~V}=9 ; \text { i.v. }=6-23)\end{array}$ & 130 \\
\hline
\end{tabular}

A infestação de 57\% em União da Vitória foi menor do que em Curitiba (97\%). Embora L. laeta tenha distribuição registrada para o sul do estado do Paraná (Marques-da-Silva e Fischer, 2005), a mesma não apareceu na amostragem deste trabalho. Loxosceles laeta é cosmopolita, contudo sua distribuição local é pequena, uma vez que em Curitiba ocorreu em apenas $6,7 \%$ das residências e mesmo em locais em que representa a única espécie de Loxosceles, como no Chile, ocorrem em apenas $29,5 \%$ das residências humanas (Schenone et al., 1970). Apesar de L. intermedia encontrar-se distribuída pela região central do município de União da Vitória, o número médio de aranhas por casa $(3,5)$ é pequeno, quando comparado com o de Curitiba $(10,8)$ (Fischer e Vasconcellos-Neto, 2005) e Chile $(8,4)$ (Schenone et al., 1970). Esse resultado pode ser interpretado de duas maneiras: ou trata-se de uma introdução devido a um processo de colonização recente ou algum fator biótico ou abiótico está limitando as populações dessas aranhas.

No ambiente urbano, $78,2 \%$ das amostras do intrae $47 \%$ do peridomicílio continham apenas uma aranha, o que suporta a hipótese de uma ocupação recente, como sugerido por Fischer e Vasconcellos-Neto (2005) para 
Curitiba. Segundo os autores, a maior presença de jovens também pode ser usada como indício de dispersão e colonização recente, uma vez que ocorreram mais jovens no intra- e no peridomicílio em meio urbano $\left(\chi_{(2)}^{2}=\right.$ $\left.15,8, \mathrm{P}<0,001 ; \chi_{(2)}^{2}=24, \mathrm{P}<0,001\right)$, em contraste com a maior freqüência de fêmeas no peridomicílio $\left(\chi_{(2)}^{2}=\right.$ $8,5, \mathrm{P}<0,05)$ e freqüências similares de jovens e fêmeas no intradomicilio em meio rural (Tabela 2).

As aranhas não apresentaram uma distribuição aleatória nos diferentes substratos, mostrando preferência por madeira, cerâmica e papel (Tabela 2), como evidenciado para L. intermedia por Fischer e Vasconcellos-Neto (2005). Esse resultado confirma que L. intermedia faz seleção entre os substratos disponíveis. É possível que o ambiente rural ofereça melhores condições de instalação para as populações, já que é costume, na região, a manutenção de madeira e produtos agrícolas em galpões e o depósito de material de construção nos quintais.

Loxosceles intermedia representou 9,5\% das aranhas amostradas no intra- $(\mathrm{N}=389)$ e $25 \%(\mathrm{~N}=$ 148) no peridomicílio da área urbana e $26,2 \%(\mathrm{~N}=133)$ no intra- e $73,8 \%(N=120)$ no peridomicilio da área rural. Logo, existem muito mais aranhas co-habitantes na área urbana, o que poderia ser um fator limitante para o crescimento populacional de L. intermedia (Fischer et al., 2006). Fischer e Krechemer (2007) verificaram que aranhas da espécie Pholcus phalangioides (Fuesslin, 1775) podem ser importantes predadores e competidores de L. intermedia. Contudo, como as teias de P. phalangioides são mais evidentes, elas são retiradas constantemente pelos moradores.

A baixa casuística de loxoscelismo no município de União da Vitória parece estar relacionada à pequena população de aranhas-marrom, principalmente no ambiente urbano. A ausência de aranhas-marrom e seus vestígios nas áreas naturais, somada a pequena quantidade de L. intermedia na área urbana, suportam a hipótese de tratar-se de uma população recente de aranhas generalistas e errantes, que encontra no ambiente antrópico substratos e condições climáticas favoráveis para sua instalação. Porém, a presença de outras espécies de aranhas pode ser um fator limitante para que as populações de L. intermedia não resultem em uma alta taxa de infestação, como ocorre em Curitiba. Assim, o manejo dos substratos preferenciais, bem como a manutenção de outras espécies de aranhas no intra- e no peridomicílio, constituem importantes atitudes preventivas de casos de loxoscelismo.

TABELA 2: Total de substratos (TS), de fêmea (F), machos (M) e jovens (J) amostrados no ambiente rural e freqüência de substratos com aranhas (\%) e total de fêmea (F), machos (M) e jovens (J) amostrados no ambiente urbano nos substratos presentes no intra- e no peridomicílio.

\begin{tabular}{llcccccccccc}
\hline \multicolumn{1}{c}{ Substrato } & \multicolumn{9}{c}{ Rural } & \multicolumn{4}{c}{ Urbano } \\
& TS & F & M & J & T & \% & F & M & J & T \\
\hline Intradomicílio & madeira & 152 & 14 & 13 & 3 & 30 & 73 & 2 & 1 & 8 & 11 \\
& papel & 23 & 4 & 3 & 4 & 11 & 27 & 1 & 0 & 0 & 1 \\
& plástico & 12 & 0 & 0 & 0 & 0 & 0 & 0 & 0 & 0 & 0 \\
& tecido & 20 & 0 & 0 & 0 & 0 & 0 & 0 & 0 & 0 & 0 \\
& partes da casa & 0 & 0 & 0 & 0 & 0 & 0 & 9 & 2 & 15 & 25 \\
& metal & 10 & 0 & 0 & 0 & 0 & 0 & 0 & 0 & 0 & 0 \\
& Total intradomicílio & 217 & 18 & 16 & 7 & 41 & - & 12 & 3 & 23 & 38 \\
& peri-madeira & 12 & 13 & 2 & 5 & 20 & 22 & 2 & 0 & 3 & 5 \\
& peri-amianto & 0 & 0 & 0 & 0 & 0 & 0 & 7 & 0 & 18 & 25 \\
& peri-cerâmica & 13 & 29 & 18 & 22 & 69 & 78 & 3 & 0 & 3 & 6 \\
& Total Peridomić́lio & 25 & 42 & 20 & 27 & 89 & - & 12 & 0 & 24 & 36 \\
& Total Geral & $\mathbf{2 4 2}$ & $\mathbf{6 0}$ & $\mathbf{3 6}$ & $\mathbf{3 4}$ & $\mathbf{1 3 0}$ & - & $\mathbf{2 4}$ & $\mathbf{3}$ & $\mathbf{4 7}$ & $\mathbf{7 4}$ \\
\hline
\end{tabular}




\section{Referências}

Fischer M. L.; Krechemer, F. S. 2007. Interações predatórias entre Pholcus phalangioides (Fuesslin) (Araneae, Pholcidae) e Loxosceles intermedia Mello-Leitão (Araneae, Sicariidae). Revista Brasileira de Zoologia, 24 (2): 474-481.

Fischer, M. L.; Vasconcellos-Neto, J. 2003. Determination of the maximum and minimum lethal temperatures (LT 50) for Loxosceles intermedia Mello-Leitão, 1934 and L. laeta (Nicolet, 1849) (Araneae, Sicariidae). Journal of Thermal Biology, 28: 563-570.

Fischer, M. L.; Vasconcellos-Neto, J. 2005. Microhabitats occupied by Loxosceles intermedia and Loxosceles laeta (Araneae: Sicariidae) in Curitiba, Paraná, Brazil. Journal of Medicinal Entomology, 42: 756-765.

Fischer, M. L.; Vasconcellos-Neto, J.; Santos-Neto, L. G. 2006. The prey and predators of Loxosceles intermedia Mello-Leitão, 1934 (Araneae; Sicariidae). Journal of Arachnology, 34: 485-488.
Gertsch, W. 1967. The spider genus Loxosceles in South America (Araneae, Scytodidae). Bulletin of the American Museum of Natural History, 136 (3): 117-174, pl. 3-11.

Hort, J. 1990. Geografia do município de União da Vitória. FAFI e UNIPORTO, União da Vitória, Brasil, 51pp.

Maack, R. 1981. Geografia Física do Paraná. J. Olympio, Rio de Janeiro, Brasil, 442pp.

Marques-da-Silva, E.; Fischer, M. L. 2005. Distribuição das espécies do gênero Loxosceles Heinecken \& Lowe, 1832 (Araneae; Sicariidae) no Estado do Paraná. Revista da Sociedade Brasileira de Medicina Tropical, 38: 331-335.

Marques-da-Silva, E.; Santos, R. S.; Fischer, M. L.; Rúbio, G. 2006. Loxosceles spider bites in the state of Paraná, Brazil: 1993-2000. Journal of Venomous Animals and Toxins, 12: 110-123.

Schenone, H.; Rojas, A.; Reves, H.; Villarroel, F.; Suarez, G. 1970. Prevalence of Loxosceles laeta in houses in Central Chile. The American Journal of Tropical Medicine and Hygiene, 19: 564567. 